\title{
Unusual case of Bilateral Angle Closure Glaucoma
}

\author{
Madhu Thapa, ' Safal Khanal', Prajwal kumar, ' Gulsan Bahadhur Shrestha, ${ }^{1}$ Ananda Kumar Sharma \\ 'B.P. Koirala Lions center for Ophthalmic studies, Institute of Medicine, Maharajgunj, Kathmandu, Nepal.
}

\begin{abstract}
Bilateral angle closure glaucoma is a rare ocular emergency which can be a potentially blinding condition. A case of 72 years male, who developed headache and vomiting after five days of demise of his mother followed by blurring of vision. He was taken to the general hospital where all the routine tests and CT Scan head failed to reveal the cause. After two days of admission, patient was referred for ophthalmic consultation. On ocular examination he was diagnosed as a case of bilateral angle closure glaucoma. After two hours of treatment patient was comfortable with normal intraocular pressure and reasonably good vision.
\end{abstract}

Keywords: Angle closure glaucoma; Intraocular pressure.

\section{INTRODUCTION}

Bilateral angle closure glaucoma is an ocular emergency in which early diagnosis with immediate intervention can have a profound effect on patient's visual outcome and ocular morbidity. Various drugs are said to be responsible for bilateral angle closure glaucoma (ACG) mainly the adrenergic drugs used locally or systemically eg ephedrine, phenylephrine, Anticholinergic drugs eg tropicamide, atropine, anti depressant like topiramate and sulfa drugs. ${ }^{1-4}$ Anatomical factor like microspherophakia, short axial length are found to be responsible for bilateral ACG. ${ }^{5}$ We present a case here who presented with bilateral angle closure glaucoma after the emotional stress of demise of his mother.

\section{CASE REPORT}

A 72 years old male presented with sudden unset of headache and ocular ache for five days, vomiting for three days and loss of vision for a day. There is no history of similar headache in the past, no history of headache with blurring of vision in the dim light, no history halos around the light, no history of trauma.
There is no history of wearing glasses, taking any ocular or systemic medication in the past. The patient had lost his mother just five days before he developed the symptoms. With the symptoms he visited a general hospital at Kathmandu where he was suspected of having some intracranial lesion and routine blood examination and CT scan head were done which all came to be normal and after two days only patient was referred for ophthalmic evaluation to our centre.

On examination, his vision was counting finger $2 \mathrm{ft}$ in both eyes, there was no improvement with pin hole. Bilaterally he had diffuse and circum corneal congestion, corneal oedema was present with epithelial microbulla, descemet folds along with endothelial dusting. Anterior chamber depth was shallow (VH grade 1) bilaterally, anterior chamber reaction could not be commented upon due to hazy cornea. Iris looked grossly normal. Lens showed NS1 in both eyes. Both the pupils were mid dilated, round and fixed (around 5-6 $\mathrm{mm}$ in both eyes). Fundus was not visible due to hazy cornea. Intra

Correspondence: Dr. Madhu Thapa, B.P. Koirala Lions center for Opthalmic studies, Institute of Medicine, Maharajgunj, Kathmandu, Nepal. Email: dr_madhut@yahoo.com, Phone: 9841236976. 
ocular pressure (IOP) were $35 \mathrm{~mm}$ of $\mathrm{Hg}$ in right eye and $36 \mathrm{~mm}$ of $\mathrm{Hg}$ in left eye.

With the findings the diagnosis of bilateral angle closure glaucoma was made and treated with Gtt Pilocarpine (2\%), one drop QID, Gtt Timolol (0.5\%) one drop BD, Gtt Betnor [Betamethasone (0.1\%) + Neomycin $(0.5 \%)$ ] one drop BE four hourly. Tab Acetazolamine $250 \mathrm{mg}$ one Tab OID, Infusion Mannitol 20\% I/V $350 \mathrm{ml}$ was given over 30 minutes.

After two hrs of treatment, Vision was 5/60, IOP was $10 \mathrm{mmHg}$ in each eye and patient was comfortable. Corneas were clear, optic disc were hyperemic bilaterally. There were glaucomfleckons seen in both the eyes. Posterior synechiae was visible 6-7 o' clock position in right and 9-10 o'clock in left eye. Gonioscopy was done which showed occludable angle in both eyes. Axial length is $21.8 \mathrm{~mm}$ in right eye and $22.17 \mathrm{~mm}$ in left eye. Center corneal thickness (CCT) $480 \mu \mathrm{m}$ and $467 \mu \mathrm{m}$; Lens diameter (anterio-posterior) were 4.97 $\mathrm{mm}$ and $4.86 \mathrm{~mm}$; Anterior chamber depth is $2.46 \mathrm{~mm}$ and $2.38 \mathrm{~mm}$; Vitreous chamber depth is $14.76 \mathrm{~mm}$ and $14.83 \mathrm{~mm}$ in right and left eye respectively.

After one day, descement folds increased than at presentation, but IOP decreased to $6 \mathrm{~mm}$ of $\mathrm{Hg}$ in both the eyes so, Tab Acetazolamide and Timolol eye drop were stopped and Pilocarpine and Betnor eye drop continued. After seven days, best corrected vision was $2 / 24$ in both the eyes and IOP was $9 \mathrm{mmHg}$ in both the eyes. Yag Peripheral iridectomy was done bilaterally. Than Pilocarpine eye drop is stopped and steroid drops tapered.

Last follow up was done after eight month of acute attack, that time vision was $6 / 24$ in right eye and $6 / 12$ in left eye, IOP was 13 in right eye and 14 in left eye. With bilateral nuclear sclerosis grade 2 in right eye and grade 1 in left eye.

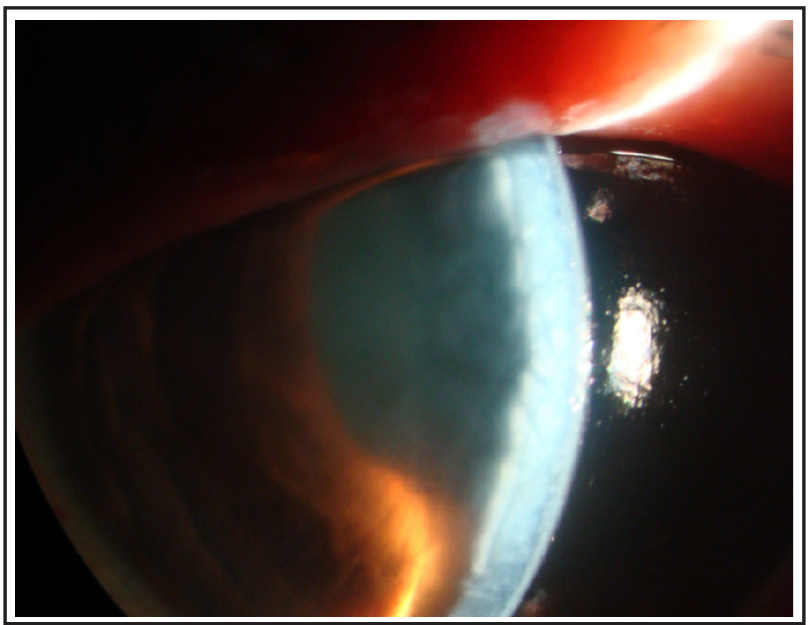

Figure 1. Corneal oedema with shallow anterior chamber in right eye.

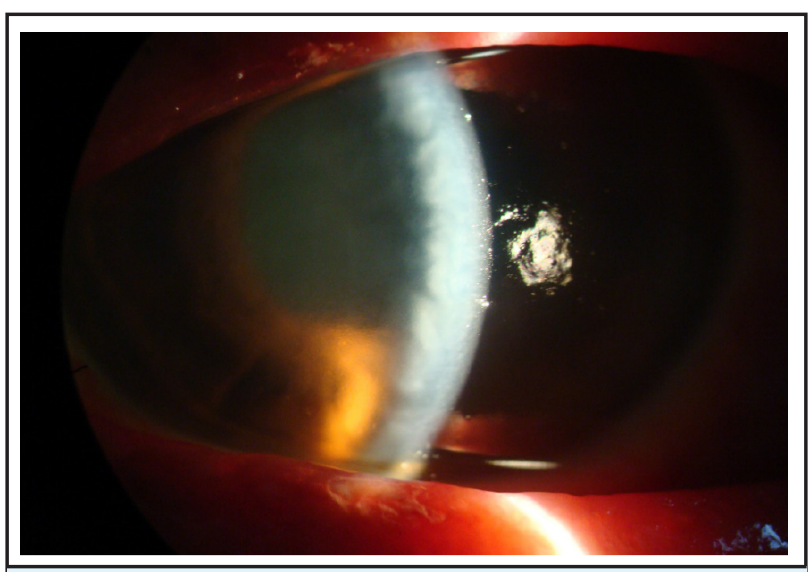

Figure 2. Corneal oedema with shallow anterior chamber in left eye.

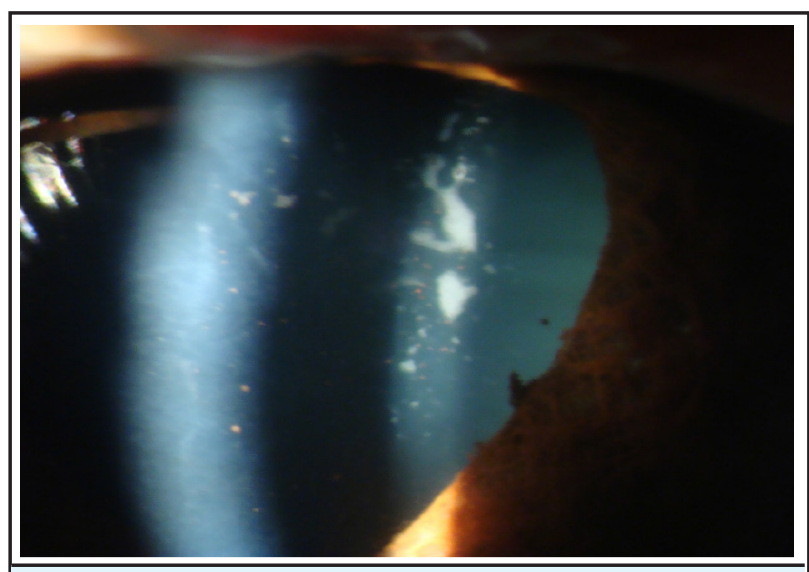

Figure 3. Glaucomflecken in right eye seen after cornea subsided.

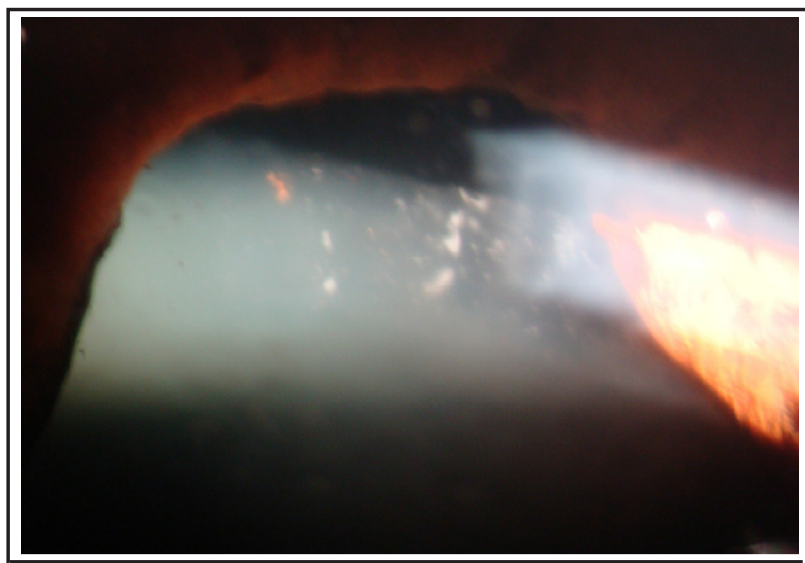

Figure 4. Glaucomfleckon seen in left eye after corneal odema subsided.

\section{DISCUSSION}

Angle closure glaucoma is one of the ocular emergencies which if not treated in time could lead to a potentially blinding condition. Simultaneous attack of angle closure glaucoma in both eyes is extremely rare. Various 
systemic and topical drugs are found to be responsible to cause bilateral angle closure attack. There are various reports which have shown that Topiramate used for depression, migraine, peripheral neuropathy, epilepsy has been proven to cause so. ${ }^{1,2}$

It can be caused by adrenergic agents, either used locally (phenylephrine drops, nasal ephedrine, or nebulized salbutamol) or systemically (epinephrine for anaphylactic shock). Drugs with anticholinergic effects including tropicamide and atropine drops can cause bilateral angle closure glaucoma as well. Literature has also shown that IV Atropine can cause Angle closure glaucoma. There are reports of bilateral angle closure glaucoma following GA administration for some non ophthalmic surgery. ${ }^{3}$

Bilateral angle closure glaucoma is reported following spinal surgery patient developed bilateral angle closure glaucoma, the pathogenesis were thought to be multifactorial: GA, prolonged prone position in patient with risk like short axial length were thought to be the risk factors. ${ }^{4}$

Sulfa-based drugs (acetazolamide, hydrochlorothiazide, cotrimoxazole) can cause acute angle closure glaucoma by ciliary body edema with anterior rotation of the iris-lens diaphragm. Iridotomy is not effective in those cases. $^{5}$
Patient with gastrointestinal infection with Camphylobacter jejuni had bilateral angle closure glaucoma where choriodal effusion was seen in USG which lead to angle closure glaucoma, which subsequently regressed after the treatment of gastroenteritis. ${ }^{6}$

Bilateral ACG is also reported in case of gaint cell arteritis. $^{7}$

Some anatomical causes like Microphthalmous, nanophthalmous, High hypermetropic patients, Microspherophakia are also thought to cause bilateral ACG. Weakness of Zonules can also lead to forward displacement of iris lens diaphragm which causes ACG. ${ }^{8}$

Literature has also shown that even after blepharoplasty, there have been incidence of angle closure glaucoma. ${ }^{9,10}$ Apart from drugs, emotion stress can cause adrenaline rush which can lead to dilatation of pupil leading to attack of ACG in high risk patient. This may be the cause in our case.

Though very rare, bilateral ACG is the potentially blinding condition if not treated in time. It not only occurs in patients with ophthalmic pathology but could be related to some non ophthalmic conditions as well. It is recommended that patient with acute headache, vomiting with ocular symptoms should be referred for ophthalmic consultation immediately to rule out bilateral ACG.

\section{REFERENCES}

1. Chalam KV, Tillis T, Syed F, Agarwal S, Brar VS. Acute bilateral simultaneous angle closure glaucoma after topiramate administration: a case report. J Med Case Reports. 2008;2:1.

2. Levy J, Yagev R, Petrava A et al. Topiramet-induced bilateral angle closure glaucoma. Can J Ophthalmology. 2006 April;41(21):221-5.

3. Ates $\mathrm{H}$ et al. Bilateral angle closure glaucoma following GA. Ophthalmology. 2001;23(3):129-30.

4. Gayat E, Gabison E, Devys M. Bilateral angle closure glaucoma after GA. Anesthesia and Analgesia. 2011 Jan;112(1):126-8.

5. Senthil S, Garudadri C, Roa HBL, Meheshwari R. Bilateral simultaneous acute angle closure caused by sulphonamide derivative: a case series. Indian Journal of ophthalmology. 2010 Nov-Dec;58(6):557-9.
6. Mukleg, S.Ramanath, Tarins. Uveal effusion association with complylobacterium jejuni infection presenting as bilateral angle closure glaucoma. J glaucoma. 2011;2.

7. Hunta TG et al. Simultaneous Bilateral angle closure glaucoma in a patient with a patient with gaint cell arteritis. J glaucoma. 2010 feb;19(2):149-50.

8. Kaushik S, Sachdev N, Pandav SS, Gupta A, Ram J. Bilateral Acute Angle closure glaucoma as a presentation of microsherophakia in adult : case report. BMC Ophthalmology. 2006;6:29.

9. Wride NK et al. Blindness after acute angle closure glaucoma after blepharoplasty. Oculoplasty reconstruction surgery. 2004 Nov;20(6):476-8.

10. Blegen I, Rade maker R, Walfs RC. Acute angle closure glaucoma after oculoplasty surgery. Orbit. 2008;127(1):49-50. 